\title{
Top-N Firewall Approximation Algorithm in Virtual Private Networks for Preserving the Dependency Relationships
}

\author{
Hayden and Beau
}

\begin{abstract}
A Virtual Private Network (VPN) is an extensively deployed network which permits roaming users to effectively access resources from the organization's network. With the aim of accessing the organization network's resources, an encrypted VPN tunnel is formulated between home network and foreign network. Even though it is helpful for the roaming users, at the same time it imposes security threats to the remote network since the remote network does not recognize what kind of data is flowing within the encrypted tunnel. All through this paper an example of IBM as home network and MSU (Michigan State University) as foreign network is taken. With the intention of addressing this issue an Xhash protocol is employed for the purpose of comparing the numbers of both parties without disclosing the number to each other. Subsequently, a firewall framework called VGuard is used that employs Xhash as the building block. A top- $N$ firewall approximation algorithm is utilized for the purpose of selecting the top- $N$ most frequently matched subset of rules from the original ruleset. The major objective is to obtain Top-N rules that cover as much traffic as possible at the same time preserving the dependency relationships. The Top- $N$ list is constructed by means of choosing the $N$ rules with highest hit-rates followed by reordering them in descending order of priority. Next, using the selected sub ruleset bootstrapping and filtering protocol is implemented.
\end{abstract}

Keywords--- Virtual Private Networks, Privacy, Network Security, Firewall Approximation.

\section{INTRODUCTION}

$\mathrm{V}$ IRTUAL Private Network (VPN) is a widely deployed technology that allows roaming users to securely use a remote computer on the public Internet as if that computer were residing on their organization's network, which henceforth allows roaming users to access some resources that are only accessible from their organization's network. VPN works in the following manner. Suppose IBM sends a field representative to one of its customers, say Michigan State

University (MSU). Assume that MSU's IP addresses are in the range 1.1.0.0-1.1.255.255 and IBM's IP addresses are in the range 2.2.0.0 to 2.2.255.255. To access resources (say a confidential customer database server with IP address 2.2.0.2) that are only accessible within IBM's network, the IBM representative uses an MSU computer (or his laptop) with an

Hayden, The University of New South Wales, Australia. Beau, Universidad de Granada, Spain.

DOI : 10.9756/BIJIEMS.8328
MSU IP address (say 1.1.0.10) to establish a secure VPN tunnel to the VPN server (with IP address 2.2.0.1) in IBM's network. Upon establishing the VPN tunnel, the IBM representative's computer is temporarily assigned a virtual IBM IP address (say 2.2.0.25).Using the VPN tunnel, the IBM representative can access any computer on the Internet as if his computer were residing on IBM's network with IP address 2.2.0.25. The payload of each packet inside the VPN tunnel is another packet (to or from the newly assigned IBM IP address 2.2.0.25), which is typically encrypted. Fig. 1 illustrates an example packet that traverses from the IBM representative's computer on MSU's network to the customer database server in IBM's network.

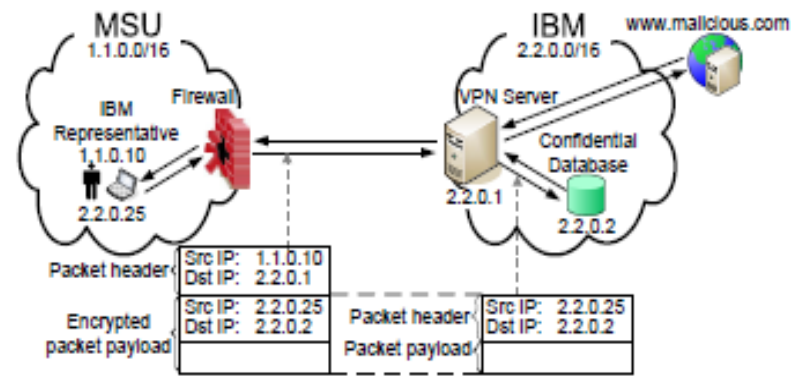

Figure 1: A Typical VPN Example

While the VPN tunnel is very useful for the IBM representative, it imposes security threats on MSU's network because MSU's firewall does not know what traffic is flowing inside the VPN tunnel. In order to address this issue VGuard, a secure and efficient framework for collaborative enforcement of firewall policies is used .In VGuard, different from CDCF, the policy owner does not know which rule matches which request; thus, it makes the selective policy updating attacks infeasible. Furthermore, unlike CDCF, VGuard obfuscates rule decisions, which prevents MSU from knowing the decision for the given packet. To make VGuard efficient, a new oblivious comparison scheme, called Xhash, is used. Also a top- $\mathrm{N}$ firewall approximation algorithm is used which selects only the top $\mathrm{N}$ firewall rules and then bootstrapping and filtering protocol is implemented.

\section{RELATED WORK}

We first discuss existing firewall frameworks and different firewall optimization strategies. The different firewall frameworks that help to preserve the privacy of the networks and the firewall optimization strategies that help to improve the packet processing throughput are discussed here. 


\section{A. Design and Implementation of Cross Domain Co- Operative Firewall}

Cross-Domain Cooperative Firewall (CDCF) [4] is a framework that allows two collaborative networks to enforce each other's firewall rules in an oblivious manner. In CDCF, when a roaming user establishes an encrypted tunnel between his home network and the foreign network, the tunnel endpoint (e.g., a VPN server) can regulate the traffic and enforce the foreign network's firewall rules, without knowing these rules. The key ingredients in CDCF are the distribution of firewall primitives across network domains, and the enabling technique of efficient oblivious membership verification.

As an example, consider the scenario in which a user roams into a foreign network $\mathrm{F}$ and establishes an encrypted tunnel with her home network H. In CDCF, each network chooses a secret key individually and never reveals it.

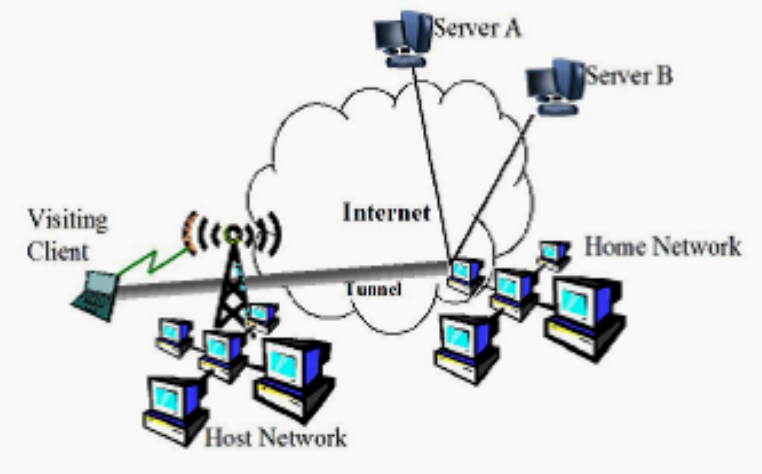

Figure 2: System Settings for Cooperative Firewall

During the bootstrapping phase, the foreign network $\mathrm{F}$ encrypts its firewall rules using its own key KF. It sends the encrypted rules to the home network $\mathrm{H}$, which applies another encryption function [9] on them with its key KH. These double-encrypted rules are then sent back to $F$. Whenever the roaming user attempts to establish a new connection within the tunnel, the VPN server at $\mathrm{H}$ encrypts the connection descriptor using $\mathrm{KH}$ and sends it to the firewall at $\mathrm{F}$, which then encrypts it again using KF. The firewall at $\mathrm{F}$ can use the oblivious membership verification algorithm to match the doubleencrypted connection descriptor against the double-encrypted rules. The resulting verdict is sent to the VPN server, which then filters the connection traffic accordingly. Note that in this process, the rule matching is done at the foreign network's firewall, while the verdict is enforced by the home network's VPN server. Such cross-domain interaction occurs only at the time of connection setup. The VPN server can cache the received verdicts and then perform the per-packet filtering task for the subsequent data packets using only local information.

\section{B. Structured Firewall Design}

A firewall is a security guard placed at the point of entry between a private network and the outside Internet such that all incoming and outgoing packets have to pass through it. The function of a firewall is to examine every incoming or outgoing packet and decide whether to accept or discard it. This function is conventionally specified by a sequence of rules, where rules often conflict. To resolve conflicts, the decision for each packet is the decision of the first rule that the packet matches. The current practice of designing a firewall directly as a sequence of rules suffers from three types of major problems:

- Consistency problem, which means that it is difficult to order the rules correctly.

- Completeness problem, which means that it is difficult to ensure thorough consideration for all types of traffic.

- Compactness problem, which means that it is difficult to keep the number of rules small

To achieve consistency, completeness and compactness a structured firewall design [5] is used which consists of two steps. First is designing firewall using Firewall Decision Diagrams (FDDs) and second is using a program that converts FDDs into compact yet functionally equivalent ones.

\section{OPTWALL: A Hierarchical Traffic-Aware Firewall}

The main objective of this paper [8] is to address the shortcomings of the firewalls and increase their ability to deal with dynamic changes in network load and topology, particularly when the network is under attack. To achieve this goal, the paper proposes a hierarchical framework for traffic aware firewall optimization. The basic tenet of this framework is that the design of next generation firewalls must leverage their packet inspection capabilities with traffic awareness in order to optimize the operational cost they incur in defending against intrusions and denial of service attacks.

In this paper, the focus is on optimizing the most widely used 'list based' firewalls. To achieve this goal a hierarchical firewall optimization approach, OPTWALL [8] to create a load balanced policy subset is used. The main challenge in the construction of these subsets stems from the need to maintain semantic integrity of the policy set at each level of the hierarchy.

The major contributions of the paper are:

- The design of OPTWALL, an adaptive hierarchical firewall optimization framework. In this framework an optimal solution to construct the hierarchy based on rule-splitting, while maintaining the integrity of the original firewall rule set is used.

- A set of heuristics, based on a trade-off between optimality,time complexity, and resource requirements to convert the list based firewall rule sets into integrity preserving hierarchical rule subsets.

- An adaptive, traffic-aware protocol to detect and defend against traffic anomalies.

- An experimental study to assess the performance of the proposed solutions and measure the impact of dynamically exploiting the traffic characteristics on the performance of firewalls.

In OPTWALL [4], the processing of a packet at a firewall starts at the root of the hierarchical structure. The packet is subsequently forwarded to the remaining levels of the hierarchy for further processing. 


\section{Traffic Aware Firewall Optimization Strategies}

List based firewalls are common type of firewalls which are used in many large networks. A firewall security is typically defined by a set of rules.A rule is a multidimensional structure, where each dimension is either a set of network fields or an action field. A network field can be source address, destination address, a service type, a protocol number, and a port number. An action field can be either accept or deny, or others. Formally, a rule $\mathrm{R}$ can be represented as: $\mathrm{R}=\left[\phi^{1}, \phi^{2}, \ldots . \phi^{\mathrm{k}} ;\right]$ where $\phi^{\mathrm{j}}$ represents network fields and $\sum$ is an action field. In an Internet environment, a typical rule can be represented as follows:

$$
<\mathrm{src}=\{\mathrm{s} 1, \mathrm{~s} 2, \ldots ., \mathrm{sn}\} ; \mathrm{dst}=\{\mathrm{d} 1, \mathrm{~d} 2, \ldots . ., \mathrm{dm}\} ;
$$$$
\operatorname{srv}=\{\sigma 1, \sigma 2, \ldots \ldots \sigma l\} ; \text { action }=\{\text { drop }\}>
$$

where 'src' represents source IP address, 'dst' represents destination IP address and 'srv' represents service type.

The process of optimization starts with the PreOptimization phase. The main objective of this phase is to remove all redundancies in the rule set. At the end of this phase, all internal and external redundancies in the rule set are removed. Unless there is a change in the current firewall policy, the pre-optimization phase is performed only once. The core component of the optimization process uses a rule set based optimizer and a traffic based optimizer. The rule based optimizer is composed of two basic components, namely the Disjoint Set Creator (DSC) and the Disjoint Set Merger (DSM). These two components are typically executed sequentially. Initially DSC detects and removes dependencies from the current rule set and makes it disjoint. The main task of DSM is to merge the rules of the disjoint rule set produced by DSC. Four schemes are used for traffic optimization.

\section{- Hot Caching}

The hot caching revolves around the concept of a hot rule set. The basic idea of this approach is to identify a small set of hot rules, relative to the original rule set, and cache these rules at the top of the rule set. It reduces overall operational cost.

\section{- Total Re-Ordering}

This is an aggressive approach where the rule set is ordered based on the current traffic characteristics. The reordering can be done using the priority of the rules.

\section{- Default Proxy}

This is a technique which creates and adds a set drop rules to the original rule set.

\section{- Online Adaptation Scheme}

Online adaptation consists of two basic schemes: profile based re-ordering and anomaly detection and countermeasure.

\section{E. Traffic Aware Top-N Firewall Ruleset Approximation Algorithm}

Packet classification is widely used in various network security and operation applications. Two of the main challenges are the increasing number of classification rules, amount of traffic and network line speed. Inorder to reduce the overhead of packet classification we need an algorithm that optimize the rule set. Such a selection algorithm [3] has the following requirements.

- Accepts rule sets with dependencies

- Suitable for online computation

- Imposes only light burden on traffic monitoring

- Dynamically adapts to traffic changes

Top-N rule set approximation algorithm is an online algorithm for obtaining a Top-N Subset of rules that satisfies the above requirements. The Top- $N$ target list is constructed by choosing the $N$ rules with highest hit-rates followed by reordering them in descending order of priority. Starting from the highest priority rule, a partition decision algorithm makes a decision for each dependent rule to either modify the rule to resolve the dependency or to include the dependent rule in the Top- $N$ list.

\section{PROBLEM STATEMENT}

\section{A. System Model}

VGuard is a secure and efficient framework for collaborative enforcement of firewall policies. In VGuard, different from CDCF [1], the policy owner does not know which rule matches which request; thus, it makes the selective policy updating attacks infeasible. VGuard obfuscates rule decisions, which prevents MSU from knowing the decision for the given packet. To make VGaurd efficient, a comparison scheme, called Xhash is used, which uses XOR and secure hash functions. Xhash is three orders of magnitude faster than the commutative encryption schemes. Moreover, VGuard uses decision diagrams to process packets, which is much faster than the linear search used in CDCF.If there are two parties, denoted MSU and IBM, where MSU has a private number N1 and IBM has a private number N2. MSU wants to compare whether N1 =N2; however, neither MSU nor IBM wants to disclose its number to others. If $\mathrm{N} 1 \neq \mathrm{N} 2$, no party should learn the value of the other party. Xhash is the oblivious comparison protocol used in the VGuard framework. Xhash works as follows:

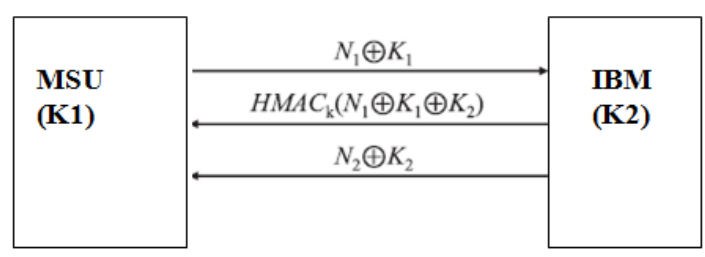

Figure 3: Xhash Protocol

If both parties have the same number bootstrapping and filtering protocol is implemented. The bootstrapping protocol is implemented as follows:

\begin{tabular}{|c|c|}
\hline MSU (K $\left.\mathbf{K}_{1}\right)$ & IBM $\left(K_{2}\right)$ \\
\hline \multicolumn{2}{|c|}{$\begin{array}{l}\text { 1. Convert firewall to non-overlapping rules. } \\
\text { 2. XOR the rules with } K_{1} \text {. } \\
\text { 3. Send the rules to IBM. }\end{array}$} \\
\hline & $\begin{array}{l}\text { 4. XOR the received rules with } \mathrm{K}_{2} \text {. } \\
\text { 5. Apply HMAC to them. } \\
\text { 6. Send resulting rules to MSU. }\end{array}$ \\
\hline
\end{tabular}

Figure 4: Bootstrapping Protocol 
The filtering protocol is implemented as follows:

MSU $\left(\mathbf{K}_{\mathbf{1}}\right) \quad$ Third Party $\left(\mathbf{K}_{\mathbf{1}}\right) \quad$ IBM $\left(\mathbf{K}_{\mathbf{2}}\right)$
1. Convert packet into 5 sequences
2. Apply XOR to every number
3. Send the resulting packet to third
Party
5. Send the resulting packet to MSU.
$\begin{aligned} & \text { 6. Make decision using decision tree. } \\ & \text { 7. Send the decision to IBM }\end{aligned}$
$\stackrel{\text { 8. Enforce the decision. }}{\longrightarrow}$

Figure 5: Filtering Protocol

Advanced firewalls, as well as intrusion detection/ prevention systems examine not only packet headers but also packet payload by checking whether its payload contains some predefined strings in a signature database. VGuard framework can be adapted to deal with the cases where MSU's firewall performs deep packet inspection. But the two main challenges in any firewall are the increasing no of firewall rules and the amount of traffic. As the no of classification rules increases, the packet classification times increases.

\section{B. Our Goal}

Our goal is to improve the packet classification throughput by selecting only the top- $\mathrm{N}$ firewall rules that are used in the VGuard framework. The existing VGuard framework focuses only on privacy preservation. Packet classification is widely used in various network security and operation applications. Two of the main challenges in the packet classification are the increasing number of classification rules and the amount of traffic. The more the number of firewall rules, the more time it will take to classify the packets. Compared to existing ones, our scheme is expected to achieve the following goals:

1. Lowers storage requirements

2. Reduces the size and power consumption of some hardware components

3. Reduces the packet classification time.

4. Preserve the privacy of the communication parties.

\section{OUR SCHEME}

\section{A. Traffic Aware Top-N Firewall Approximation Algorithm}

The proposed system is the VGuard framework that uses a top- $\mathrm{N}$ approximation on firewall rules. The basic idea is to dynamically select a subset of $\mathrm{N}$ rules (Top- $\mathrm{N}$ ) from the original rule set with the highest hit-rates based on traffic pattern.

Initially Xhash protocol is used to check whether two communication parties are having same number without disclosing it to each other. Once the number matches, there will be the original rule set from which the policy owner selects only top-N sub rules. The rules with higher hit rate have to be selected and arrange those rules in the decreasing order of priority. Rules that are independent of others (i.e. no conflict), are safely included. Rules that only depend on other target rules can also be included, as long as their relative order is preserved in the resulting list. For target rules that depend on at least one non-target rule, either resolves the conflicts with the dependent rules or include the dependent rules to the top- $\mathrm{N}$ list, retaining their relative priorities. Conflicts can be resolved by splitting the target rule concerned into smaller derived rules that are disjoint with the dependent rules.

\section{B. Top-N target List}

A hit-rate table is used to maintain the hit-rate of the firewall rules. By sorting the hit-rate table in descending order of hit-rates, the first $\mathrm{N}$ rules are the target rules and together it is called the target set. Since some of the target rules may depend on rules that are not in the target set, one cannot simply select the $\mathrm{N}$ target rules as the top- $\mathrm{N}$ rules.

\section{Dependency Graph}

A dependency graph is used to represent the dependency relationships among rules in a rule set. A dependency graph is a directed graph with a vertex vi representing the rule ri for each rule in the rule set and an edge ec,d that points from a vertex with a larger ID c to another vertex with a smaller ID d where rc depends on rd. Moreover, the graph is acyclic because, by definition, only lower priority rules can depend on higher priority rules

\section{Algorithm 1: Dependency Graph Construction}

1. Input:

A rule set with priority

2. Output:

A set of dependency graph $\mathrm{G}=(\mathrm{V}, \mathrm{E})$

3. procedure Dependency graph Construction

4. Initialize a vertex vi for all ri

5. for each pair of rules (vi, vj) do

6. if overlap ((vi, vj)) and $\mathrm{p}(\mathrm{ri}) \neq \mathrm{p}(\mathrm{rj})$ then

7. add an edge eij from vj to vi

8. w (eij ) $\leftarrow$ no. of derived rules

To construct a dependency graph, the matching spaces of rules are pair-wisely compared. Two rules that have overlapping matching spaces and differ in actions are connected by an edge. The weight of the edge w (eij) represents the minimum number of derived rules of ri that results after disjointing ri and rj.

\section{E. Top- $N$ Selection}

Top-N selection is a process to select up to $\mathrm{N}$ rules in order to maximize the overall hit-rate. The hit-rate of a rule is the fraction of packet classification queries that the rule has provided an action for. Rules that are independent of others (i.e. no conflict), are safely included. Rules that only depend on other target rules can also be included, as long as their relative order is preserved in the resulting list. For target rules that depend on at least one non-target rule, we need to either resolve the conflicts with the dependent rules or include the dependent rules to the top- $\mathrm{N}$ list, retaining their relative priorities 


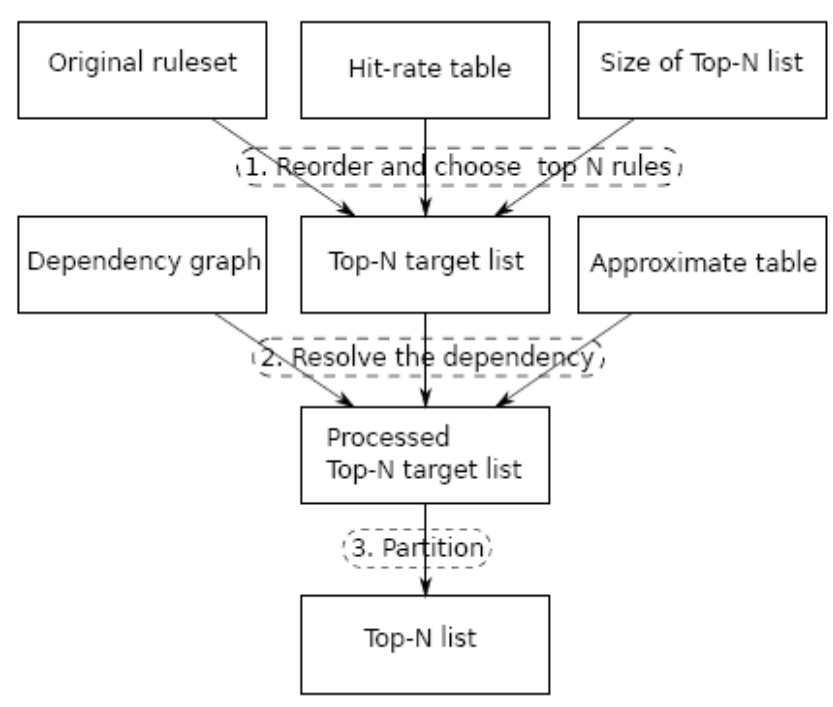

Figure 6: Top-N Approximation Algorithm

The above fig shows the working of Top- $\mathrm{N}$ firewall Approximation Algorithm.

Top- $\mathrm{N}$ approximation algorithm has the following steps, illustrated in Figure 6. The Top-N target list is constructed by choosing the $\mathrm{N}$ rules with highest hit-rates followed by reordering them in descending order of priority. 2) A dependency sub-graph rooted at the first rule in the target list is obtained. Starting from the root, the partition decision algorithm (Algorithm 3) makes a decision of either partitioning the target rule or including the dependent rule in the Top-N list, for each dependent rule in the sub-graph. After the above two steps, each target rule has a list of dependent rules RP to resolve conflicts and another list of dependent rules $\mathrm{RD}$ to be included together in the top- $\mathrm{N}$ list. Step 3, initializes an empty top- $\mathrm{N}$ list and iteratively processes the target rules in descending order of the hit-rates. Using conflict resolution algorithms such as [6], each target rule is partitioned to resolve conflicts with RP, if any. The resulting derived rules and $\mathrm{RD}$ are added to the top-N list.

F. Algorithm 2: Top-N Selection

1. procedure TOP-N SELECTION

2. select $\mathrm{N}$ target rules with highest reference

3. sort them to descending priority

4. for each target rule ri do

5. $\mathrm{T}=$ partition_decision $(\mathrm{ri}, \mathrm{G})$

6. if Top-N list is full then

7. Stop and output the Top-N list

8. else

9. put $\mathrm{T}$ into Top-N list

\section{G. Algorithm 3: Partition Decision}

1. procedure PARTITION DECISION

2. for each rule rj that ri depends on do

3. if rj disjoints with all the other rules that ri depends on and size (sub tree rooted at rj $) \geq v($ eij) then

4. decision $\leftarrow$ PARTITION

5. for each group do

6. $\mathrm{S} \leftarrow$ all rules in this group

7. $\mathrm{sD} \leftarrow 0$. total number of derived rules
8. $\quad$ rk $\leftarrow$ getfirst(S)

9. $\mathrm{sD} \leftarrow \mathrm{sD}+\mathrm{v}($ eik $)$

10. if size(rk) $\geq \mathrm{sD}$ then

11. decision $\leftarrow$ PARTITION

12. $\mathrm{T} \leftarrow$ derived rules

13. else

14. decision $\leftarrow$ KEEP

15. $\mathrm{T} \leftarrow$ all rules in the dependency graph root at rk

16. while $\mathrm{S}$ is not empty do

17. $\mathrm{Q} \leftarrow$ all rules in $\mathrm{S}$ and overlap with $\mathrm{rk}$

18. for each rule $r k \in Q$ do

19. sl $\leftarrow$ lookup $(\mathrm{rk}, \mathrm{rl})$, lookup the approximation table

20. if size(rk) $\geq$ sl then

21. decision $\leftarrow$ PARTITION

22. $\mathrm{T} \leftarrow$ derived rules

23. else

24. decision $\leftarrow$ KEEP

25. $\mathrm{T} \leftarrow$ all rules root at rk Frep

26. return $\mathrm{T}$

Using the top- $\mathrm{N}$ rules the bootstrapping protocol and filtering protocol is implemented. Also a deep packet inspection using VGuard framework is also done.

\section{CONCLUSION}

In this paper, a firewall approximation algorithm is proposed that is used to select only the top- $\mathrm{N}$ rules that covers as much traffic as possible. This is an online adaptation algorithm which selects the top- $\mathrm{N}$ rules with higher hit rate based on the traffic changes before bootstrapping protocol is implemented.

Our scheme has several following advantages:

1. The VGuard framework along with the top-N firewall approximation algorithm helps to preserve the privacy of communication parties in a VPN.

2. The proposed system not only focuses on privacy preservation but also on traffic characteristics.

3. The overall packet classification throughput can be increased by selecting only the top- $\mathrm{N}$ rules.

4. This is an online adaptation algorithm which works based on the traffic changes.

5. It lowers the storage requirements since only $\mathrm{N}$ rules with highest hit rate are selected.

\section{REFERENCES}

[1] S. Acharya, M. Abliz, B. Mills, T.F. Znati, J. Wang, Z. Ge and A. Greenberg, “OPTWALL: A hierarchical traffic-aware firewall”, Proc. of the 12th Annual Network and Distributed System Security Symposium, 2005.

[2] S. Charya, J. Wang, Z. Ge, T. Znati and A. Greenberg, "Traffic aware firewall optimization strategies", IEEE International Conference on Communications, Vol. 5, 2006

[3] P. Brucker, "On the complexity of clustering problems", Optimization and Operations Research, Springer-Verlag, Pp. 45-54, 1977, 1997.

[4] Y.K. Chang, "Fast Binary and Multiway Prefix Searches for Packet Forwarding”, Computer Networks, Vol. 51, No. 3, Pp. 588-605, 2007.

[5] J. Cheng, H. Yang, S.H. Wong and S. Lu, "Design and Implementation of Cross-Domain Cooperative Firewall”, Proc.IEEE Int'l Conf. Network Protocols (ICNP), 2007.

[6] M.G. Gouda and A.X. Liu, "Structured Firewall Design", Computer Networks J., Vol. 51, No. 4, Pp. 1106-1120, 2007. 
[7] P. Gupta and N. McKeown, "Algorithms for Packet Classification," IEEE Network, Vol. 15, No. 2, Pp. 24-32, 2001.

[8] H. Hamed and E. Al-Shaer, "Dynamic rule-ordering optimization for high-speed firewall filtering”, Proc. of the 2006 ACM Symp. on Inform., comput. and commun. Security, 2006.

[9] A.X. Liu, E. Torng and C.R. Meiners, "Firewall compressor: An algorithm for minimizing firewall policies", Proc. Of the 27th Conf. on Comp. Comm, 2008.

[10] S.C. Pohlig and M.E. Hellman, "An Improved Algorithm for Computing Logarithms over GF(p) and Its Cryptographic Significance”, IEEE Trans. Information and System Security, Pp. 106-110, 1978.

[11] S. Singh, F. Baboesu, G. Varghese and J. Wang, "Packet classification using multidimensional cutting”, SIGCOMM, 2003. 